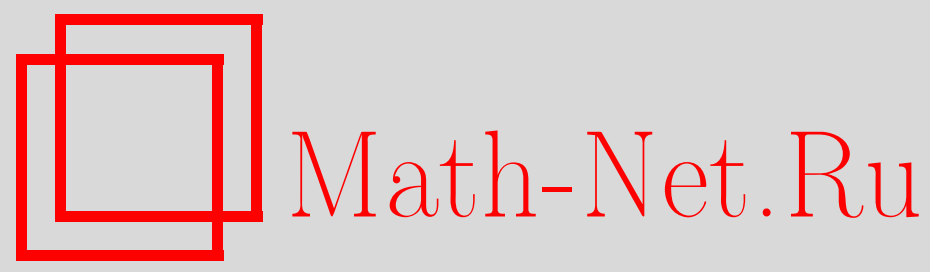

П. Г. Кононенко, Аффинные типы $L$-многогранников 5мерных решеток, Матем. заметки, 2002, том 71, выпуск $3,412-430$

DOI: https://doi.org/10.4213/mzm356

Использование Общероссийского математического портала Math-Net.Ru подразумевает, что вы прочитали и согласны с пользовательским соглашением http://www.mathnet.ru/rus/agreement

Параметры загрузки:

IP: 52.90 .164 .192

26 апреля 2023 г., 13:59:21 
УДК $514.174 .6+519.112 .7$

\title{
АФФИННЫЕ ТИПЫ $L$-МНОГОГРАННИКОВ 5-МЕРНЫХ РЕШЕТОК
}

\section{П. Г. Кононенко}

\begin{abstract}
Построен алгоритм вывода всех афффинно неэквивалентных видов $L$-многогранников $n$-мерных решеток при $n \leqslant 5$. Вычислительная часть алгоритма, предназначенная для вычисления на ПК, основана на связи геометрии решеток и теории гиперметрических пространств. Впервые получен полный список аффинных типов (139 типов) $L$-многогранников 5 -мерных решеток.
\end{abstract}

Библиография: 14 названий.

1. Введение. $L$-многогранником, или многогранником Делоне, $n$-мерной точечной решетки назьвается вьпуклый $n$-мерньй многогранник, все вершины которого расположены на поверхности сферы, причем замкнутьй шар, ограниченньй этой сферой, не содержит других точек решетки. Множество всех $L$-многогранников данной решетки образует нормальное разбиение (разбиение "грань в грань") пространства $E^{n}$, комбинаторно и метрически двойственное разбиению на области Дирихле-Вороного для точек этой решетки. Такая двойственность позволяет, зная строение одного из этих разбиений, немедленно получить и строение второго. Результаты относительно строений названных разбиений и их многогранников используются в задачах о параллелоэдрах, о наиболее экономных решетчатых покрытиях и в ряде других задач геометрии решеток.

Одной из задач относительно $L$-разбиений $n$-мерных решеток является отыскание при данном $n$ всех аффинных типов $L$-многогранников. Для случаев $n=2$ и $n=3$ эти типы давно и широко известны (см., например, [1]), при $n=4$ они были получены в 1987 году С. С. Рышковьп и Р. Эрдалом [2]; на другом пути вывод 4-мерных $L$-многогранников сделан в статьях [3], [4]. Для больших размерностей вопрос о перечислении типов $L$-многогранников оставался открытым, хотя при $n=5$ частичное решение задачи дано в заметке [5] и намечен путь к полному решению в работе [4].

В этой статье завершено решение задачи об аффинных типах $L$-многогранников 5 -мерных решеток, а именно доказана следующая теорема.

ТЕОрема 1. Существует всего 139 видов аффинно неәквивалентных L-многогранников 5-мерных решеток, строение 138 из которых дано в таблице 1. Не названным в таблице L-многогранником является симплекс двойного обтема.

Доказательство теоремы 1, помимо идей из статьи [4], существенно опирается на методы теории гиперметрик, связь которой с теорией $L$-многогранников была обнаружена в [6], [7]. На последнем этапе доказательство теоремы 1 явилось следствием нахождения всех попарно неэквивалентных граней гиперметрического конуса $H Y P_{n+1}$ при $n=5$, за исключением граней, порождающих решетку меньшей размерности чем $n=5$. Тем самым, доказана 
ТАБЛИЦА 1

Аффинные типы 5-мерных $L$-многогранников

\begin{tabular}{|c|c|c|c|c|c|c|}
\hline 1 & 2 & 3 & 4 & 5 & строение & гиперграни \\
\hline 1 & 6 & 6 & 15 & 1 & $S^{5}$ & $6 S^{4}$ \\
\hline 2 & 7 & 12 & 14 & 2 & & $12 S^{4}$ \\
\hline 3 & 7 & 10 & 14 & 1 & $p\left(V_{2,2}^{4}\right)$ & $1 V_{2,2}^{4}, 9 S^{4}$ \\
\hline 4 & 7 & 7 & 14 & 1 & $p\left(p\left(p\left(P^{2}\right)\right)\right)$ & $3 p\left(p\left(P^{2}\right)\right), 4 S^{4}$ \\
\hline 5 & 8 & 13 & 13 & 2 & & $3 p\left(p\left(P^{2}\right)\right), 1 V_{2,2}^{4}, 9 S^{4}$ \\
\hline 6 & 8 & 14 & 13 & 2 & & $4 p\left(p\left(P^{2}\right)\right), 10 S^{4}$ \\
\hline 7 & 8 & 10 & 13 & 1 & & $4 p\left(p\left(P^{2}\right)\right), 2 V_{2,2}^{4}, 4 S^{4}$ \\
\hline 8 & 8 & 15 & 13 & 1 & & $3 p\left(p\left(P^{2}\right)\right), 12 S^{4}$ \\
\hline 9 & 8 & 7 & 13 & 1 & $p\left(p\left(C^{1} \times S^{2}\right)\right)$ & $2 p\left(C^{1} \times S^{2}\right), 3 p\left(p\left(P^{2}\right)\right), 2 S^{4}$ \\
\hline 10 & 8 & 9 & 13 & 1 & $p\left(S_{4}\right)$ & $1 S_{4}, 4 p\left(p\left(P^{2}\right)\right), 4 S^{4}$ \\
\hline 11 & 8 & 10 & 13 & 1 & $p\left(p\left(C^{3}\right)\right)$ & $2 p\left(C^{3}\right), 8 S^{4}$ \\
\hline 12 & 8 & 8 & 13 & 1 & & $8 p\left(p\left(P^{2}\right)\right)$ \\
\hline 13 & 9 & 14 & 12 & 2 & & $2 p\left(C^{3}\right), 3 V_{2,2}^{4}, 9 S^{4}$ \\
\hline 14 & 9 & 13 & 12 & 2 & & $2 S_{4}, 5 p\left(p\left(P^{2}\right)\right), 6 S^{4}$ \\
\hline 15 & 9 & 13 & 12 & 2 & & $2 p\left(C^{1} \times S^{2}\right), 5 p\left(p\left(P^{2}\right)\right), 1 V_{2,2}^{4}, 5 S^{4}$ \\
\hline 16 & 9 & 12 & 12 & 1 & & $12 p\left(p\left(P^{2}\right)\right)$ \\
\hline 17 & 9 & 15 & 12 & 2 & & $9 p\left(p\left(P^{2}\right)\right), 6 S^{4}$ \\
\hline 18 & 9 & 10 & 12 & 1 & & $3 S_{4}, 3 p\left(p\left(P^{2}\right)\right), 1 V_{2,2}^{4}, 3 S^{4}$ \\
\hline 19 & 9 & 10 & 12 & 1 & $p\left(S_{6}\right)$ & $1 S_{6}, 1 p\left(C^{3}\right), 4 p\left(p\left(P^{2}\right)\right), 4 S^{4}$ \\
\hline 20 & 9 & 13 & 12 & 1 & & $1 S_{4}, 8 p\left(p\left(P^{2}\right)\right), 4 S^{4}$ \\
\hline 21 & 9 & 7 & 12 & 1 & $p\left(C^{1} \times S^{3}\right)$ & $1 C^{1} \times S^{3}, 4 p\left(C^{1} \times S^{2}\right), 2 S^{4}$ \\
\hline 22 & 9 & 8 & 12 & 1 & $p\left(S_{5}\right)$ & $1 S_{5}, 2 p\left(C^{1} \times S^{2}\right), 4 p\left(p\left(P^{2}\right)\right), 1 S^{4}$ \\
\hline 23 & 9 & 9 & 12 & 1 & & $2 p\left(C^{1} \times S^{2}\right), 2 S_{4}, 3 p\left(p\left(P^{2}\right)\right), 2 S^{4}$ \\
\hline 24 & 9 & 14 & 12 & 1 & & $2 p\left(C^{3}\right), 4 p\left(p\left(P^{2}\right)\right), 8 S^{4}$ \\
\hline 25 & 9 & 17 & 12 & 1 & $p\left(C^{4}\right)$ & $1 C^{4}, 16 S^{4}$ \\
\hline 26 & 10 & 17 & 11 & 2 & & $1 C^{4}, 6 V_{2,2}^{4}, 10 S^{4}$ \\
\hline 27 & 10 & 15 & 11 & 2 & & $2 p\left(C^{3}\right), 2 S_{4}, 5 p\left(p\left(P^{2}\right)\right), 6 S^{4}$ \\
\hline 28 & 10 & 13 & 11 & 2 & & $1 S_{6}, 1 p\left(C^{3}\right), 2 S_{4}, 3 p\left(p\left(P^{2}\right)\right), 1 V_{2,2}^{4}, 5 S^{4}$ \\
\hline 29 & 10 & 12 & 11 & 2 & & $1 S_{5}, 2 p\left(C^{1} \times S^{2}\right), 2 S_{4}, 4 p\left(p\left(P^{2}\right)\right), 3 S^{4}$ \\
\hline 30 & 10 & 11 & 11 & 1 & & $4 p\left(C^{1} \times S^{2}\right), 2 S_{4}, 5 p\left(p\left(P^{2}\right)\right)$ \\
\hline 31 & 10 & 11 & 11 & 1 & & $1 C^{1} \times S^{3}, 4 p\left(C^{1} \times S^{2}\right), 4 p\left(p\left(P^{2}\right)\right), 2 V_{2,2}^{4}$ \\
\hline 32 & 10 & 15 & 11 & 2 & & $6 p\left(C^{1} \times S^{2}\right), 3 p\left(p\left(P^{2}\right)\right), 6 S^{4}$ \\
\hline 33 & 10 & 8 & 12 & 1 & $p\left(p\left(P^{3}\right)\right)$ & $2 p\left(P^{3}\right), 6 p\left(p\left(P^{2}\right)\right)$ \\
\hline 34 & 10 & 10 & 11 & 1 & & $2 S_{6}, 2 S_{4}, 4 p\left(p\left(P^{2}\right)\right), 2 S^{4}$ \\
\hline 35 & 10 & 12 & 11 & 1 & & $5 S_{4}, 5 p\left(p\left(P^{2}\right)\right), 2 S^{4}$ \\
\hline 36 & 10 & 17 & 11 & 1 & & $1 C^{4}, 8 p\left(p\left(P^{2}\right)\right), 8 S^{4}$ \\
\hline 37 & 10 & 10 & 11 & 1 & & $2 S_{6}, 4 p\left(C^{1} \times S^{2}\right), 4 S^{4}$ \\
\hline 38 & 10 & 12 & 11 & 1 & & $1 S_{6}, 1 p\left(C^{3}\right), 2 p\left(C^{1} \times S^{2}\right), 6 p\left(p\left(P^{2}\right)\right), 2 S^{4}$ \\
\hline 39 & 10 & 7 & 11 & 1 & $p\left(T_{6}\right)$ & $1 T_{6}, 6 p\left(C^{1} \times S^{2}\right)$ \\
\hline 40 & 10 & 7 & 11 & 1 & $C^{1} \times S^{4}$ & $5 C^{1} \times S^{3}, 2 S^{4}$ \\
\hline 41 & 10 & 8 & 11 & 1 & & $1 C^{1} \times S^{3}, 2 S_{5}, 2 p\left(C^{1} \times S^{2}\right), 3 p\left(p\left(P^{2}\right)\right)$ \\
\hline 42 & 10 & 9 & 11 & 1 & & $2 S_{5}, 2 p\left(C^{1} \times S^{2}\right), 2 S_{4}, 1 p\left(p\left(P^{2}\right)\right), 2 S^{4}$ \\
\hline
\end{tabular}




\begin{tabular}{|c|c|c|c|c|c|c|}
\hline 1 & 2 & 3 & 4 & 5 & строение & гиперграни \\
\hline 43 & 10 & 10 & 11 & 1 & $p\left(S_{8}\right)$ & $1 S_{8}, 1 p\left(C^{1} \times S^{2}\right), 2 p\left(C^{3}\right), 3 p\left(p\left(P^{2}\right)\right), 3 S^{4}$ \\
\hline 44 & 10 & 12 & 11 & 2 & & $2 S_{6}, 8 p\left(p\left(P^{2}\right)\right), 2 S^{4}$ \\
\hline 45 & 10 & 16 & 11 & 1 & & $4 p\left(C^{3}\right), 4 p\left(p\left(P^{2}\right)\right), 8 S^{4}$ \\
\hline $46^{*}$ & 10 & 32 & 11 & 1 & $C^{5}$ & $32 S^{4}$ \\
\hline 47 & 11 & 17 & 10 & 2 & & $1 C^{4}, 4 S_{4}, 4 p\left(p\left(P^{2}\right)\right), 1 V_{2,2}^{4}, 7 S^{4}$ \\
\hline 48 & 11 & 14 & 10 & 2 & & $2 S_{6}, 2 p\left(C^{3}\right), 2 S_{4}, 4 p\left(p\left(P^{2}\right)\right), 4 S^{4}$ \\
\hline 49 & 11 & 12 & 10 & 1 & & $4 S_{6}, 4 p\left(p\left(P^{2}\right)\right), 2 V_{2,2}^{4}, 2 S^{4}$ \\
\hline 50 & 11 & 12 & 10 & 2 & & $\begin{array}{l}1 S_{8}, 1 S_{6}, 1 p\left(C^{1} \times S^{2}\right), 2 S_{4}, 1 p\left(C^{3}\right) \\
\quad 3 p\left(p\left(P^{2}\right)\right), 3 S^{4}\end{array}$ \\
\hline 51 & 11 & 11 & 10 & 1 & & $2 S_{6}, 1 S_{5}, 2 p\left(C^{1} \times S^{2}\right), 2 S_{4}, 3 p\left(p\left(P^{2}\right)\right), 1 S^{4}$ \\
\hline 52 & 11 & 14 & 10 & 2 & & $2 S_{5}, 1 S_{6}, 2 p\left(C^{1} \times S^{2}\right), 1 p\left(C^{3}\right), 4 p\left(p\left(P^{2}\right)\right), 4 S^{4}$ \\
\hline 53 & 11 & 10 & 10 & 1 & & $1 C^{1} \times S^{3}, 2 S_{5}, 4 p\left(C^{1} \times S^{2}\right), 2 S_{4}, 1 p\left(p\left(P^{2}\right)\right)$ \\
\hline 54 & 11 & 10 & 10 & 1 & & $2 S_{6}, 8 p\left(C^{1} \times S^{2}\right)$ \\
\hline 55 & 11 & 13 & 11 & 1 & $p\left(b p\left(P^{3}\right)\right)$ & $1 b p\left(P^{3}\right), 12 p\left(p\left(P^{2}\right)\right)$ \\
\hline 56 & 11 & 8 & 11 & 1 & $p\left(C^{1} \times p\left(P^{2}\right)\right)$ & $1 C^{1} \times p\left(P^{2}\right), 1 p\left(P^{3}\right), 4 p\left(C^{1} \times S^{2}\right), 2 p\left(p\left(P^{2}\right)\right)$ \\
\hline 57 & 11 & 11 & 11 & 1 & & $2 p\left(P^{3}\right), 3 S_{4}, 6 p\left(p\left(P^{2}\right)\right)$ \\
\hline 58 & 11 & 10 & 10 & 1 & & $1 S_{8}, 1 S_{5}, 2 S_{6}, 1 p\left(C^{1} \times S^{2}\right), 3 p\left(p\left(P^{2}\right)\right), 2 S^{4}$ \\
\hline 59 & 11 & 8 & 10 & 1 & & $1 T_{6}, 1 C^{1} \times S^{3}, 3 S_{5}, 2 p\left(C^{1} \times S^{2}\right), 1 S^{4}$ \\
\hline 60 & 11 & 11 & 10 & 2 & & $5 S_{5}, 5 p\left(p\left(P^{2}\right)\right), 1 S^{4}$ \\
\hline 61 & 11 & 17 & 10 & 1 & & $1 C^{4}, 2 p\left(C^{1} \times S^{2}\right), 2 p\left(C^{3}\right), 6 p\left(p\left(P^{2}\right)\right), 6 S^{4}$ \\
\hline 62 & 11 & 11 & 10 & 1 & & $\begin{array}{l}1 S_{8}, 1 C^{1} \times S^{3}, 3 p\left(C^{1} \times S^{2}\right), 2 p\left(C^{3}\right) \\
\quad 3 p\left(p\left(P^{2}\right)\right), 1 S^{4}\end{array}$ \\
\hline 63 & 11 & 11 & 10 & 1 & $p\left(S_{11}\right)$ & $1 S_{11}, 5 p\left(C^{3}\right), 5 S^{4}$ \\
\hline 64 & 11 & 16 & 10 & 2 & & $1 S_{6}, 5 p\left(C^{3}\right), 4 p\left(p\left(P^{2}\right)\right), 6 S^{4}$ \\
\hline 65 & 12 & 13 & 10 & 1 & & $1 b p\left(P^{3}\right), 6 S_{4}, 6 p\left(p\left(P^{2}\right)\right)$ \\
\hline 66 & 12 & 12 & 10 & 1 & & $2 p\left(P^{3}\right), 2 S_{6}, 4 p\left(C^{1} \times S^{2}\right), 4 p\left(p\left(P^{2}\right)\right)$ \\
\hline $67^{*}$ & 12 & 20 & 10 & 1 & & $20 V_{2,2}^{4}$ \\
\hline 68 & 12 & 17 & 9 & 2 & & $2 S_{6}, 1 C^{4}, 2 p\left(C^{3}\right), 2 S_{4}, 4 p\left(p\left(P^{2}\right)\right), 6 S^{4}$ \\
\hline 69 & 12 & 15 & 9 & 2 & & $1 S_{8}, 2 S_{6}, 1 p\left(C^{1} \times S^{2}\right), 4 p\left(C^{3}\right), 2 p\left(p\left(P^{2}\right)\right), 5 S^{4}$ \\
\hline 70 & 12 & 12 & 9 & 1 & & $6 S_{6}, 6 p\left(p\left(P^{2}\right)\right)$ \\
\hline 71 & 12 & 11 & 9 & 1 & & $2 S_{8}, 2 S_{6}, 2 p\left(C^{1} \times S^{2}\right), 2 S_{4}, 1 p\left(p\left(P^{2}\right)\right), 2 S^{4}$ \\
\hline 72 & 12 & 10 & 9 & 1 & & $1 S_{8}, 1 C^{1} \times S^{3}, 2 S_{5}, 2 S_{6}, 3 p\left(C^{1} \times S^{2}\right), 1 S^{4}$ \\
\hline 73 & 12 & 11 & 10 & 1 & $C^{1} \times V_{2,2}^{4}$ & $9 C^{1} \times S^{3}, 2 V_{2,2}^{4}$ \\
\hline $74^{*}$ & 12 & 24 & 10 & 1 & $b p\left(b p\left(P^{3}\right)\right)$ & $24 p\left(p\left(P^{2}\right)\right)$ \\
\hline 75 & 12 & 13 & 10 & 1 & & $1 b p\left(P^{3}\right), 8 p\left(C^{1} \times S^{2}\right), 4 p\left(p\left(P^{2}\right)\right)$ \\
\hline 76 & 12 & 8 & 10 & 1 & $C^{1} \times p\left(p\left(P^{2}\right)\right)$ & $2 C^{1} \times p\left(P^{2}\right), 4 C^{1} \times S^{3}, 2 p\left(p\left(P^{2}\right)\right)$ \\
\hline 77 & 12 & 8 & 10 & 1 & & $2 C^{1} \times p\left(P^{2}\right), 2 S_{5}, 4 p\left(C^{1} \times S^{2}\right)$ \\
\hline 78 & 12 & 10 & 10 & 1 & & $\begin{array}{l}1 C^{1} \times p\left(P^{2}\right), 1 p\left(P^{3}\right), 2 S_{5}, 2 S_{4}, 2 p\left(C^{1} \times S^{2}\right) \\
\quad 2 p\left(p\left(P^{2}\right)\right)\end{array}$ \\
\hline 79 & 12 & 17 & 9 & 2 & & $4 S_{5}, 1 C^{4}, 8 p\left(p\left(P^{2}\right)\right), 4 S^{4}$ \\
\hline 80 & 12 & 12 & 9 & 2 & & $1 S_{11}, 3 S_{6}, 2 p\left(C^{3}\right), 3 p\left(p\left(P^{2}\right)\right), 3 S^{4}$ \\
\hline 81 & 12 & 7 & 9 & 1 & & $4 T_{6}, 3 C^{1} \times S^{3}$ \\
\hline 82 & 12 & 10 & 9 & 1 & & $1 T_{6}, 3 S_{8}, 3 p\left(C^{1} \times S^{2}\right), 3 S^{4}$ \\
\hline 83 & 12 & 13 & 9 & 2 & & $2 S_{8}, 2 S_{5}, 2 p\left(C^{3}\right), 5 p\left(p\left(P^{2}\right)\right), 2 S^{4}$ \\
\hline
\end{tabular}




\begin{tabular}{|c|c|c|c|c|c|c|}
\hline 1 & 2 & 3 & 4 & 5 & строение & гиперграни \\
\hline 84 & 12 & 9 & 9 & 1 & & $3 C^{1} \times S^{3}, 6 S_{5}$ \\
\hline 85 & 12 & 19 & 9 & 2 & & $1 C^{4}, 8 p\left(C^{3}\right), 10 S^{4}$ \\
\hline 86 & 12 & 10 & 9 & 1 & & $4 S_{8}, 6 p\left(p\left(P^{2}\right)\right)$ \\
\hline 87 & 12 & 17 & 9 & 1 & & $1 C^{1} \times S^{3}, 2 C^{4}, 4 p\left(C^{1} \times S^{2}\right), 6 p\left(p\left(P^{2}\right)\right), 4 S^{4}$ \\
\hline 88 & 13 & 13 & 9 & 1 & & $1 b p\left(P^{3}\right), 2 S_{5}, 2 S_{6}, 2 p\left(C^{1} \times S^{2}\right), 2 S_{4}, 4 p\left(p\left(P^{2}\right)\right)$ \\
\hline 89 & 13 & 11 & 9 & 1 & & $\begin{array}{l}1 C^{1} \times p\left(P^{2}\right), 1 p\left(P^{3}\right), 1 S_{8}, 2 S_{6}, 1 S_{5} \\
\quad 3 p\left(C^{1} \times S^{2}\right), 2 p\left(p\left(P^{2}\right)\right)\end{array}$ \\
\hline 90 & 13 & 8 & 10 & 1 & $p\left(C^{1} \times C^{1} \times S^{2}\right)$ & $1 C^{1} \times C^{1} \times S^{2}, 3 p\left(P^{3}\right), 4 p\left(C^{1} \times S^{2}\right)$ \\
\hline 91 & 13 & 19 & 8 & 2 & & $3 S_{6}, 2 C^{4}, 3 p\left(C^{3}\right), 3 p\left(p\left(P^{2}\right)\right), 8 S^{4}$ \\
\hline 92 & 13 & 11 & 8 & 1 & & $1 S_{11}, 2 S_{8}, 3 S_{6}, 2 p\left(C^{1} \times S^{2}\right), 3 S^{4}$ \\
\hline 93 & 13 & 9 & 9 & 1 & & $2 C^{1} \times p\left(P^{2}\right), 1 T_{6}, 4 S_{5}, 2 p\left(C^{1} \times S^{2}\right)$ \\
\hline 94 & 13 & 11 & 10 & 1 & $p\left(C^{1} \times C^{3}\right)$ & $1 C^{1} \times C^{3}, 8 p\left(C^{1} \times S^{2}\right), 2 p\left(C^{3}\right)$ \\
\hline 95 & 13 & 10 & 8 & 1 & & $4 S_{8}, 2 C^{1} \times S^{3}, 2 S_{5}, 2 S^{4}$ \\
\hline 96 & 13 & 14 & 8 & 2 & & $1 S_{11}, 2 S_{8}, 5 p\left(C^{3}\right), 3 p\left(p\left(P^{2}\right)\right), 3 S^{4}$ \\
\hline 97 & 13 & 17 & 8 & 2 & & $2 S_{8}, 1 S_{5}, 1 C^{4}, 4 p\left(C^{3}\right), 4 p\left(p\left(P^{2}\right)\right), 5 S^{4}$ \\
\hline 98 & 14 & 13 & 9 & 1 & & $1 b p\left(P^{3}\right), 4 p\left(P^{3}\right), 8 p\left(C^{1} \times S^{2}\right)$ \\
\hline 99 & 14 & 13 & 8 & 1 & & $1 b p\left(P^{3}\right), 2 S_{8}, 4 S_{6}, 2 p\left(C^{1} \times S^{2}\right), 4 p\left(p\left(P^{2}\right)\right)$ \\
\hline 100 & 14 & 8 & 9 & 1 & $C^{1} \times p\left(C^{1} \times S^{2}\right)$ & $\begin{array}{l}1 C^{1} \times C^{1} \times S^{2}, 3 C^{1} \times p\left(P^{2}\right), 2 C^{1} \times S^{3} \\
\quad 2 p\left(C^{1} \times S^{2}\right)\end{array}$ \\
\hline 101 & 14 & 9 & 9 & 1 & & $\begin{array}{l}1 C^{1} \times C^{1} \times S^{2}, 1 C^{1} \times p\left(P^{2}\right), 2 p\left(P^{3}\right), 4 S_{5}, \\
\quad 1 p\left(p\left(P^{2}\right)\right)\end{array}$ \\
\hline 102 & 14 & 10 & 9 & 1 & $C^{1} \times S_{4}$ & $4 C^{1} \times p\left(P^{2}\right), 4 C^{1} \times S^{3}, 2 S_{4}$ \\
\hline $103^{*}$ & 14 & 18 & 9 & 1 & & $18 S_{4}$ \\
\hline 104 & 14 & 10 & 8 & 1 & & $2 C^{1} \times p\left(P^{2}\right), 4 S_{8}, 4 p\left(C^{1} \times S^{2}\right)$ \\
\hline 105 & 14 & 11 & 9 & 1 & & $1 C^{1} \times C^{3}, 4 S_{5}, 2 S_{6}, 4 p\left(C^{1} \times S^{2}\right)$ \\
\hline $106 *$ & 14 & 20 & 9 & 1 & $b p\left(C^{1} \times C^{3}\right)$ & $16 p\left(C^{1} \times S^{2}\right), 4 p\left(C^{3}\right)$ \\
\hline 107 & 14 & 11 & 9 & 1 & $C^{1} \times p\left(C^{3}\right)$ & $1 C^{1} \times C^{3}, 8 C^{1} \times S^{3}, 2 p\left(C^{3}\right)$ \\
\hline 108 & 14 & 20 & 7 & 2 & & $2 S_{8}, 3 C^{4}, 4 p\left(C^{3}\right), 3 p\left(p\left(P^{2}\right)\right), 8 S^{4}$ \\
\hline 109 & 14 & 11 & 7 & 1 & & $2 S_{11}, 4 S_{8}, 1 C^{1} \times S^{3}, 4 S^{4}$ \\
\hline 110 & 14 & 17 & 7 & 2 & & $2 S_{11}, 1 C^{4}, 8 p\left(C^{3}\right), 6 S^{4}$ \\
\hline 111 & 15 & 13 & 8 & 1 & & $\begin{array}{l}1 C^{1} \times p\left(P^{2}\right), 2 b p\left(P^{3}\right), 1 p\left(P^{3}\right), 4 S_{5} \\
\quad 4 p\left(C^{1} \times S^{2}\right), 1 p\left(p\left(P^{2}\right)\right)\end{array}$ \\
\hline 112 & 15 & 8 & 8 & 1 & & $1 C^{1} \times C^{1} \times S^{2}, 3 C^{1} \times p\left(P^{2}\right), 4 T_{6}$ \\
\hline 113 & 15 & 11 & 8 & 1 & & $1 C^{1} \times C^{1} \times S^{2}, 3 p\left(P^{3}\right), 4 S_{8}, 3 p\left(p\left(P^{2}\right)\right)$ \\
\hline 114 & 15 & 11 & 8 & 1 & & $1 C^{1} \times C^{3}, 1 T_{6}, 3 S_{8}, 3 S_{5}, 3 p\left(C^{1} \times S^{2}\right)$ \\
\hline 115 & 15 & 12 & 6 & 1 & & $6 S_{11}, 6 S^{4}$ \\
\hline 116 & 15 & 22 & 6 & 2 & & $1 S_{11}, 5 C^{4}, 5 p\left(C^{3}\right), 11 S^{4}$ \\
\hline 117 & 16 & 14 & 7 & 1 & & $3 b p\left(P^{3}\right), 4 S_{8}, 4 p\left(C^{1} \times S^{2}\right), 3 p\left(p\left(P^{2}\right)\right)$ \\
\hline 118 & 16 & 11 & 8 & 1 & $C^{1} \times S_{6}$ & $1 C^{1} \times C^{3}, 4 C^{1} \times p\left(P^{2}\right), 4 C^{1} \times S^{3}, 2 S_{6}$ \\
\hline 119 & 16 & 8 & 8 & 1 & $C^{1} \times C^{1} \times S^{3}$ & $4 C^{1} \times C^{1} \times S^{2}, 4 C^{1} \times S^{3}$ \\
\hline 120 & 16 & 9 & 8 & 1 & $C^{1} \times S_{5}$ & $2 C^{1} \times C^{1} \times S^{2}, 4 C^{1} \times p\left(P^{2}\right), 2 S_{5}, 1 C^{1} \times S^{3}$ \\
\hline $121^{*}$ & 16 & 16 & 8 & 1 & & $8 S_{5}, 8 S_{6}$ \\
\hline 122 & 16 & 11 & 7 & 1 & & $1 C^{1} \times C^{3}, 2 S_{11}, 4 S_{8}, 4 p\left(C^{1} \times S^{2}\right)$ \\
\hline $123^{*}$ & 16 & 18 & 8 & 1 & $C^{1} \times C^{4}$ & $16 C^{1} \times S^{3}, 2 C^{4}$ \\
\hline
\end{tabular}




\begin{tabular}{||r|r|r|r|r||c|l||}
\hline 1 & 2 & 3 & 4 & 5 & строение & \multicolumn{1}{|c||}{ гиперграни } \\
\hline 124 & 16 & 26 & 5 & 2 & & $10 C^{4}, 16 S^{4}$ \\
125 & 17 & 9 & 9 & 1 & $p\left(P^{4}\right)$ & $1 P^{4}, 8 p\left(P^{3}\right)$ \\
$126^{*}$ & 18 & 16 & 8 & 1 & $b p\left(P^{4}\right)$ & $16 p\left(P^{3}\right)$ \\
127 & 18 & 9 & 8 & 1 & $C^{1} \times p\left(P^{3}\right)$ & $1 P^{4}, 6 C^{1} \times p\left(P^{2}\right), 2 p\left(P^{3}\right)$ \\
128 & 18 & 8 & 7 & 1 & $C^{1} \times T_{6}$ & $6 C^{1} \times C^{1} \times S^{2}, 2 T_{6}$ \\
129 & 18 & 11 & 7 & 1 & $C^{1} \times S_{8}$ & $1 C^{1} \times C^{1} \times S^{2}, 2 C^{1} \times C^{3}, 3 C^{1} \times p\left(P^{2}\right), 2 S_{8}$, \\
& & & & & & $3 C^{1} \times S^{3}$ \\
130 & 18 & 11 & 7 & 1 & & $3 C^{1} \times C^{3}, 8 T_{6}$ \\
$131^{*}$ & 18 & 14 & 7 & 1 & & $2 T_{6}, 12 S_{8}$ \\
$132^{*}$ & 20 & 14 & 7 & 1 & $C^{1} \times b p\left(P^{3}\right)$ & $12 C^{1} \times p\left(P^{2}\right), 2 b p\left(P^{3}\right)$ \\
133 & 20 & 9 & 7 & 1 & $C^{1} \times C^{1} \times p\left(P^{2}\right)$ & $1 P^{4}, 4 C^{1} \times C^{1} \times S^{2}, 4 C^{1} \times p\left(P^{2}\right)$ \\
134 & 20 & 12 & 6 & 1 & $C^{1} \times S_{11}$ & $5 C^{1} \times C^{3}, 2 S_{11}, 5 C^{1} \times S^{3}$ \\
$135^{*}$ & 20 & 12 & 6 & 1 & & $12 S_{11}$ \\
136 & 24 & 9 & 6 & 1 & $C^{1} \times C^{1} \times C^{1} \times S^{2}$ & $3 P^{4}, 6 C^{1} \times C^{1} \times S^{2}$ \\
$137^{*}$ & 24 & 12 & 6 & 1 & $C^{1} \times C^{1} \times C^{3}$ & $8 C^{1} \times C^{1} \times S^{2}, 4 C^{1} \times C^{3}$ \\
$138^{*}$ & 32 & 10 & 5 & 1 & $P^{5}$ & $10 P^{4}$ \\
\hline
\end{tabular}

ТЕорема 2. Количества классов әквивалентности (относительно перестановок множества $X=\{0, \ldots, n\})$ граней конуса $H Y P_{6}=C_{6}$, порождающих решетку невырожденной размерности $n=5$, распределенные по убыванию размерности граней, образуют строку $(4,26,131,490,1179,1893,1995,1410,647,170,0,0,0,0)$.

2. Гиперметрический конус, cut-конус и $L$-многогранники решеток. Приведенные здесь определения и результаты относительно гиперметрического конуса и cut-конуса, если для них не указаны другие литературные источники, взяты из работ М. Деза [8] и М. Деза, В.П. Гришухина и М. Лоран [7], [9], [10].

Для натурального числа $n$ рассмотрим конечное множество $X=\{0,1, \ldots, n\}$. Дuстаниионной функиией называется отображение $d: X \times X \rightarrow \mathbb{R}_{+}$, удовлетворяющее для всех $i, j \in X$ соотношениям $d(i, j)=d(j, i), d(i, i)=0$. Пара $(X, d)$ называется дистанционным пространством.

Положив $d_{i j}=d(i, j)$ для всех $i, j \in X$, мы отождествим любое из пространств $(X, d)$ с точкой $d=\left(d_{01}, d_{02}, \ldots, d_{n-1, n}\right)$ в пространстве $\mathbb{R}^{N}=\mathbb{R}^{n(n+1) / 2}$ (здесь и далее, $N=n(n+1) / 2)$.

Дистанционное пространство $(X, d)$ назьвается гиперметрическим (соответственно векторно-гиперметрическим), если дистанционная функция $d$ удовлетворяет гиперметрическим (соответственно векторно-гиперметрическим) неравенствам

$$
\sum_{0 \leqslant i<j \leqslant n} x^{i} x^{j} d_{i j} \leqslant 0
$$

для всех $\boldsymbol{x}=\left(x^{0}, x^{1}, \ldots, x^{n}\right) \in \mathbb{Z}^{n+1}$ при условии $\sum_{0 \leqslant i \leqslant n} x^{i}=1$ (соответственно $\left.\sum_{0 \leqslant i \leqslant n} x^{i}=0\right)$.

Множество гиперметрических пространств (соответственно векторно-гиперметрических) образует в пространстве $\mathbb{R}^{N}$ вьпукльй замкнутый конус $H Y P_{n+1}$ (соответственно $N E G_{n+1}$ ). Конус $H Y P_{n+1}$ (в отличие от конуса $N E G_{n+1}$ ) является полиэдральным [10]. 
Пусть $S \subset X=\{0,1, \ldots, n\}, S \neq \varnothing, X$. Дистанционное пространство $(X, d)$ назьвается cut-метрикой (метрикой Хәмминга), если дистанционная функция $d=d^{S}$ определяется соотношением

$$
d_{i j}^{S}= \begin{cases}1, & \text { если }|S \cap\{i, j\}|=1, \\ 0 & \text { в противном случае. }\end{cases}
$$

Cut-конусом $C_{n+1}$ в пространстве $\mathbb{R}^{N}$ называется коническая оболочкамножества всех cut-метрик $d^{S} \in \mathbb{R}^{N}$ :

$$
C_{n+1}=\left\{d=\sum_{S \subset X} \lambda_{S} d^{S} \in \mathbb{R}^{N} \mid \lambda_{S} \geqslant 0\right\}
$$

Поскольку для каждой порождающей конус $C_{n+1}$ cut-метрики $d^{S}$ левая часть гиперметрического неравенства (1) при $x \in \mathbb{Z}^{n+1}, \sum_{0 \leqslant i \leqslant n} x^{i}=1$, принимает значение $x(S)(1-x(S)) \leqslant 0$, где $x(S)=\sum_{i \in S} x^{i}$, то $C_{n+1} \subseteq H Y P_{n+1}$.

Таким образом, для конусов $C_{n+1}, H Y P_{n+1}$ при всех $n$ имеют место включения $C_{n+1}$ $\subseteq H Y P_{n+1}$. При $n \leqslant 5$ имеют место равенства $C_{n+1}=H Y P_{n+1}(n \leqslant 4$ [7], $n=5[11])$. Для $n=6$ включение строгое: $C_{n+1} \subset H Y P_{n+1}$.

Любая гиперметрика $d \in H Y P_{n+1}$ имеет реализацию в $k$-мерном евклидовом пространстве $E^{k}(k \leqslant n)$, порождая $L$-многогранник $P_{d}$ точечной решетки $L_{d}$. А именно, существует отображение $\phi: X=\{0, \ldots, n\} \rightarrow E^{k}, \phi(i)=A_{i} \in E^{k}(i=0, \ldots, n)$, где $k=\operatorname{dim}\left\{A_{0}, \ldots, A_{n}\right\}$, причем

1) $d_{i j}={\overline{A_{i} A_{j}}}^{2}(0 \leqslant i<j \leqslant n)$;

2) система векторов $\bar{v}_{i}=\bar{A}_{0} A_{i}(i=0, \ldots, n)$, определенная с точностью до движения в $E^{k}$, имеет квадратную матрицу попарных скалярных произведений $a_{i j}=\bar{v}_{i} \bar{v}_{j}$ $(1 \leqslant i, j \leqslant n)$, связанную с гиперметрикой $d \in H Y P_{n+1}$ однозначными соотношениями

$$
a_{i i}=d_{0 i}, \quad 1 \leqslant i \leqslant n, \quad a_{i j}=\frac{1}{2}\left(d_{0 i}+d_{0 j}-d_{i j}\right), \quad 1 \leqslant i<j \leqslant n,
$$

или обратными соотношениями

$$
d_{0 i}=a_{i i}, \quad 1 \leqslant i \leqslant n, \quad d_{i j}=a_{i i}+a_{j j}-2 a_{i j}, \quad 1 \leqslant i<j \leqslant n ;
$$

3 ) векторы $\bar{v}_{1}, \ldots, \bar{v}_{n}$ порождают в $E^{k} k$-мерную точечную решетку

$$
L_{d}=\left\{T \in E^{k} \mid \overline{A_{0} T}=\sum_{1 \leqslant i \leqslant n} x^{i} \bar{v}_{i}, x=\left(x^{1}, \ldots, x^{n}\right) \in \mathbb{Z}^{n}\right\}
$$

4) существует $k$-мерная сфера $S_{d} \subset E^{k}$, содержашая на поверхности точки $A_{0}, \ldots, A_{n}$ и не содержашая точек решетки внутри, т.е. выпуклая оболочка точек $L_{d} \cap S_{d}$ образует $L$-многогранник решетки $L_{d}$;

$5)$ если обозначить $\bar{c}$ - радиус-вектор центра сферы $S_{d}$, то для всякого набора:

a) $\boldsymbol{x}=\left(x^{0}, \ldots, x^{n}\right) \in \mathbb{Z}^{n+1}$ c $\sum_{i=0}^{n} x^{i}=1$,

b) $\boldsymbol{y} \in \mathbb{Z}^{n+1} \mathrm{c} \sum_{i=0}^{n} y^{i}=0$, 
обратная величина в левой части:

a) гиперметрического,

b) векторно-гиперметрического

неравенства (1) принимает вид

$$
\begin{aligned}
\text { a) } \sum_{0 \leqslant i<j \leqslant n} x^{i} x^{j} d_{i j} & =\sum_{1 \leqslant i, j \leqslant n} x^{i} x^{j} a_{i j}-\sum_{1 \leqslant i \leqslant n} x^{i} a_{i i} \\
& =\left\|\sum_{1 \leqslant i \leqslant n} x^{i} \bar{v}_{i}-\bar{c}\right\|^{2}-\|\bar{c}\|^{2} ; \\
\text { b) }-\sum_{0 \leqslant i<j \leqslant n} y^{i} y^{j} d_{i j} & =\sum_{1 \leqslant i, j \leqslant n} y^{i} y^{j} a_{i j}=\left\|\sum_{1 \leqslant i \leqslant n} y^{i} \bar{v}_{i}\right\|^{2} ;
\end{aligned}
$$

и имеет геометрический смысл в $E^{k}$ :

a) степени точки относительно сферы $S_{d}$,

b) квадрата длины вектора.

С другой стороны, любой $L$-многогранник $P$ решетки $L$ вместе с отображением $\phi$ из $X=\{0, \ldots, n\}$ во множество вершин многогранника $P$ порождает гиперметрику $d \in H Y P_{n+1}$, определенную соотношениями $d_{i j}=\|\phi(i)-\phi(j)\|^{2}(0 \leqslant i<j \leqslant n)$.

Таким образом, сушествует взаимно однозначное соответствие между

а) гиперметриками $(X, d),|X|=n+1$,

б) парами $(P, \phi)$, где $\phi$ - отображение из $X=\{0, \ldots, n\}$ во множество вершин

$L$-многогранника $P$ решетки $L$, причем множество $\phi(X)$ определяет решетку $L$.

На множестве вектор-строк из $\mathbb{Z}^{n+1}$ введем обозначение $\boldsymbol{u}_{i}=\left(u_{i}^{0}, \ldots, u_{n}^{0}\right), u_{i}^{i}=1$ и $u_{i}^{j}=0$ при $i \neq j$. Следуя работе [7], введем пару дуальных друг другу понятий аннулятора гиперметрики

$$
\operatorname{Ann}(d):=\left\{\boldsymbol{x} \in \mathbb{Z}^{n+1} \backslash\left\{\boldsymbol{u}_{0}, \ldots, \boldsymbol{u}_{n}\right\} \mid \sum_{i=0}^{n} x^{i}=1, \sum_{0 \leqslant i<j \leqslant n} x^{i} x^{j} d_{i j}=0\right\}
$$

и гиперплоскости $H_{x}$ в гиперметрическом пространстве $\mathbb{R}^{N}$, определенной для каждого $\boldsymbol{x} \in \mathbb{Z}^{n+1} \backslash\left\{\boldsymbol{u}_{0}, \ldots, \boldsymbol{u}_{n}\right\}$ с $\sum_{i=0}^{n} x^{i}=1$ соотношением

$$
H_{\boldsymbol{x}}=\left\{d=\left(d_{i j}\right)_{0 \leqslant i<j \leqslant n} \in \mathbb{R}^{n(n+1) / 2} \mid \sum_{0 \leqslant i<j \leqslant n} x^{i} x^{j} d_{i j}=0\right\} .
$$

Очевидно, что $\boldsymbol{x} \in \operatorname{Ann}(d) \Longleftrightarrow d \in H_{\boldsymbol{x}}$ (для соответствующих $\boldsymbol{x}$ и $d$ ).

Теперь каждой точке $T$ решетки $L_{d}$, определенной гиперметрикой $d$, поставим в соответствие множество

$$
Z(T)=\left\{\boldsymbol{x}=\left(x^{0}, \ldots, x^{n}\right) \in \mathbb{Z}^{n+1} \mid \sum_{0 \leqslant i \leqslant n} x^{i}=1, \bar{t}=\sum_{0 \leqslant i \leqslant n} x^{i} \bar{v}_{i}\right\}
$$

где $\bar{v}_{0}=\mathbf{0}$, а $\bar{t}$ - радиус-вектор точки $T$. Принимая во внимание выражение (7), заключаем, что для множества вершин $\operatorname{ext} P_{d} L$-многогранника $P_{d}$ справедливо

$$
\operatorname{Ann}(d) \cup\left\{\boldsymbol{u}_{0}, \ldots, \boldsymbol{u}_{n}\right\}=\bigcup_{T \in \operatorname{ext} P_{d}} Z(T) .
$$


С другой стороны, рассматривая конус $H Y P_{n+1}$, обозначим через

$$
F_{\boldsymbol{x}}=H Y P_{n+1} \cap H_{\boldsymbol{x}}=\left\{d \in H Y P_{n+1} \mid \sum_{0 \leqslant i \leqslant n} x^{i} x^{j} d_{i j}=0\right\}
$$

грань конуса $H Y P_{n+1}$, содержащуюся в гиперплоскости $H_{\boldsymbol{x}}$, а для каждой гиперметрики $d$, лежащей на поверхности конуса $H Y P_{n+1}$, через $F(d)$ обозначим грань наименьшей размерности, содержащую $d$, т.е.

$$
F(d)=\bigcap_{d \in H_{\boldsymbol{x}}} F_{\boldsymbol{x}}=\bigcap_{x \in \operatorname{Ann}(d)} F_{\boldsymbol{x}}
$$

Полиэдральность конуса гиперметрик $H Y P_{n+1}$ (доказанная в [9]), заданного бесконечной системой неравенств $(1)$, означает существование эквивалентной ей конечной подсистемы неравенств, соответствующей тем точкам $\boldsymbol{x} \in \mathbb{Z}^{n+1}$ с $\sum_{i=0}^{n} x^{i}=1$, для которых множество $H_{\boldsymbol{x}} \cap H Y P_{n+1}$ является гипергранью конуса $H Y P_{n+1}$. Назовем эти точки минимальными заслоняющими.

Множество минимальных заслоняющих точек для $n \leqslant 5$ найдено П. Ассуа [6], a paнее, но в иной интерпретации, Е.П. Барановским [12]. Барановский [13] указал также минимальные заслоняющие точки для случая $n=6$.

Перечислим минимальные заслоняющие точки для $n \leqslant 5$ (с точностью до перестановки элементов вектор-строки):

$$
\begin{aligned}
& n=2: \quad(-1,1,1) \\
& n=3: \quad(-1,1,1,0) \\
& n=4: \quad(-1,1,1,0,0),(-1,-1,1,1,1) \\
& n=5: \quad(-1,1,1,0,0,0),(-1,-1,1,1,1,0),(-2,-1,1,1,1,1),(-1,-1,-1,2,1,1) .
\end{aligned}
$$

3. Невырождденные гиперметрики. Пользуясь обозначениями и результатами предыдущего пункта и рассматривая соотношение (10), заметим, что при нахождении строения $L$-многогранника $P_{d}$, соответствуюшего гиперметрике $d \in H Y P_{n+1}$, достаточно знать и на практике наиболее удобно рассматривать только те гиперметрики, для которых представление любой точки $T \in E^{k}$ во множестве $Z(T)$ единственно.

Равенство $k=\operatorname{rank}\left(a_{i j}\right)_{1 \leqslant i, j \leqslant n}=\operatorname{rank}\left\{\bar{v}_{i}\right\}_{1 \leqslant i \leqslant n}=\operatorname{dim}\left(L_{d}\right)$, справедливое для любой гиперметрики $d \in H Y P_{n+1}$, позволяет сформулировать следующее определение.

ОПРЕДЕЛЕНИЕ. Гиперметрика $d \in H Y P_{n+1}$ назьвается невырожденной, если выполнено равенство

$$
k \equiv \operatorname{rank}\left(a_{i j}\right)_{1 \leqslant i, j \leqslant n} \equiv \operatorname{rank}\left\{\bar{v}_{i}\right\}_{1 \leqslant i \leqslant n} \equiv \operatorname{dim}\left(L_{d}\right)=n
$$

где $\left(a_{i j}\right)_{1 \leqslant i, j \leqslant n}=a=\alpha(d)$, а векторы $\bar{v}_{1}, \ldots, \bar{v}_{n}$ определены в п. 2 .

Наряду с гиперплоскостями (8) будем рассматривать в пространстве $\mathbb{R}^{n(n+1) / 2}$ гиперплоскости $H_{\boldsymbol{y}}$, соответствующие точкам $\boldsymbol{y} \in \mathbb{R}^{n+1}$ таким, что $\boldsymbol{y} \in \mathbb{Z}^{n+1} \backslash\{\mathbf{0}\}$ и $\sum_{i=0}^{n} y^{i}=0$. 
ОПРЕДЕЛЕНИЕ. Дефектом гиперметрики $d \in H Y P_{n+1}$ назьвается множество

$$
\begin{aligned}
\mathrm{K}(d) & =\left\{\boldsymbol{y} \in \mathbb{Z}^{n+1} \mid \sum_{i=0}^{n} y^{i}=0, \sum_{0 \leqslant i<j \leqslant n} y^{i} y^{j} d_{i j}=0\right\} \\
& =\{\boldsymbol{0}\} \cup\left\{\boldsymbol{y}=\left(y^{0}, \ldots, y^{n}\right) \in \mathbb{Z}^{n+1} \backslash\{\mathbf{0}\} \mid \sum_{i=0}^{n} y^{i}=0, d \in H_{\boldsymbol{y}}\right\} \\
& =\left\{\boldsymbol{y}=\left(y^{0}, \ldots, y^{n}\right) \in \mathbb{Z}^{n+1} \mid \sum_{i=0}^{n} y^{i}=0, \sum_{i=0}^{n} y^{i} \bar{v}_{i}=\mathbf{0}\right\} .
\end{aligned}
$$

Относительно приведенных вьше определений результаты п. 2 позволяют сформулировать следующие леммы.

Лемма 1. Невырожденность гиперметрики $d \in H Y P_{n+1}$ әквивалентна каждому из следующих утверждений:

1) $\mathrm{K}(d)=\mathbf{0}$

2) система точек $\phi(X)=\left\{A_{0}, \ldots, A_{n}\right\}$ определяет основной симплекс решетки $L_{d}$, а вектор-строки множества $\operatorname{Ann}(d) \cup\left\{\boldsymbol{u}_{0}, \ldots, \boldsymbol{u}_{n}\right\}$ представляют собой баричентрические координаты вершин многогранника $P_{d}$ относительно барицентрического базиса $\left\{A_{0}, \ldots, A_{n}\right\}$.

ЛЕмма 2. Существует взаимно однозначное соответствие мехду

а) невырохденными гиперметриками $(X, d),|X|=n+1$;

б) парами $(P, \phi)$, где $P$ - это $L$-многогранник некоторой $n$-мерной решетки $L$, а $\phi$-инвективное отображсение из $X=\{0,1, \ldots, n\}$ во мнохсество вершин многогранника $P$, причем $\phi(X)$ образует основной симплекс решетки $L$.

4. Размерность решеток и аффинные типы $L$-многогранников, соответствующие граням конусов $H Y P_{n+1}$ и $C_{n+1}$. Рассмотрим проекцию $\pi: \mathbb{Z}^{n+1} \rightarrow \mathbb{Z}^{n}$, $\pi:\left(x^{0}, x^{1}, \ldots, x^{n}\right) \mapsto\left(x^{1}, \ldots, x^{n}\right)$. Полученные проектированием множества Ann* $(d):=$ $\pi(\operatorname{Ann}(d)), \mathrm{K}^{*}(d):=\pi(\mathrm{K}(d))$ будем, как и исходные, называть соответственно аннулятором и дефектом гиперметрики $d$.

Очевидно, что $\mathrm{K}^{*}(d)$ имеет и следующее представление:

$$
\mathrm{K}^{*}(d)=\left\{\boldsymbol{y}=\left(y^{1}, \ldots, y^{n}\right) \in \mathbb{Z}^{n} \mid \sum_{1 \leqslant i \leqslant n} y^{i} \bar{v}_{i}=\mathbf{0}\right\} .
$$

Заметим, что для любого $\boldsymbol{x} \in \mathbb{Z}^{n+1}$ с $\sum_{i=0}^{n} x^{i}=1$ гиперплоскость $H_{\boldsymbol{x}}$ делит пространство $\mathbb{R}^{n(n+1) / 2}$ на два полупространства, замькание одного из которых содержит конусы $C_{n+1}$ и $H Y P_{n+1}$ (поскольку $C_{n+1} \subseteq H Y P_{n+1}$ ). Аналогичное утверждение выполнено и для гиперплоскости $H_{\boldsymbol{y}}$ при любом $\boldsymbol{y} \in \mathbb{Z}^{n+1} \backslash\{\mathbf{0}\}$ с $\sum_{i=0}^{n} y^{i}=0$, поскольку $C_{n+1} \subseteq H Y P_{n+1} \subseteq N E G_{n+1}$.

Отсюда следует, что аннулятор $\operatorname{Ann}(d)$ и дефект $\mathrm{K}(d)$ сохраняются для всех точек $d$ из относительной внутренности ri $F$ грани $F$ (т.е. множества внутренних точек для $F$ в aффинном подпространстве $\operatorname{aff}(F)$, натянутом на множество точек грани $F$ ). Это позволяет ввести следующие определения. 
ОПРЕДЕЛЕНИЕ. Пусть $F$ - грань конуса $H Y P_{n+1}$ (или $C_{n+1}$ ).

a) Аннулятором грани $F$ будем назьвать множество $\operatorname{Ann}(F)=\operatorname{Ann}(d)$ (и множество $\left.\operatorname{Ann}^{*}(F)=\operatorname{Ann}^{*}(d)\right)$ для любой $d \in \operatorname{ri} F$.

б) Дефектом грани $F$ будем называть множество $\mathrm{K}(F)=\mathrm{K}(d)$ (и множество $\mathrm{K}^{*}(F)$ $\left.=\mathrm{K}^{*}(d)\right)$ для любой $d \in \operatorname{ri} F$.

ОПРеДЕЛЕнИЕ. Грань $F$ конуса $C_{n+1}$ (или $H Y P_{n+1}$ ) называется невырожденной, если $\mathrm{K}^{*}(F)=\mathbf{0}$, т.е. все гиперметрики $d \in \operatorname{ri} F$ из относительной внутренности грани $F$ невырождены.

Для произвольной гиперметрики $d \in H Y P_{n+1}$ рассмотрим линейное отображение $\omega_{d}: \mathbb{Z}^{n} \rightarrow E^{k}$, определенное соотношением

$$
\omega_{d}: \boldsymbol{x}=\left(x^{1}, \ldots, x^{n}\right) \mapsto \omega_{d}(\boldsymbol{x})=\sum_{1 \leqslant i \leqslant n} x^{i} \bar{v}_{i}
$$

где $\bar{v}_{1}, \ldots, \bar{v}_{n}$ - векторы, определенные для гиперметрики $d$ в п. 2 . Очевидно, что $\operatorname{Ker}\left(\omega_{d}\right)$ $=\mathrm{K}^{*}(d)$ и $\operatorname{Im}\left(\omega_{d}\right)=L_{d}$, где $L_{d}$ определена для $d$ соотношением (6).

Отображение $\omega_{d}$ порож дает семейство естественных изоморфизмов $\widehat{\omega}_{d}: \mathbb{Z}^{n} / \mathrm{K}^{*}(F) \rightarrow$ $L_{d}$ для всех $d$ из относительной внутренности некоторой грани $F$ конуса $H Y P_{n+1}$ (или $\left.C_{n+1}\right)$. Принимая во внимание, что множество вершин ext $P_{d}$ многогранника $P_{d}$ представимо в следующем виде: $\operatorname{ext} P_{d}=\omega\left(\operatorname{Ann}(d) \cup\left\{\boldsymbol{u}_{0}, \ldots, \boldsymbol{u}_{n}\right\}\right)$, получаем следующую лемму.

Лемма 3. Пусть $F$ - грань конуса $H Y P_{n+1}\left(\right.$ или $\left.C_{n+1}\right)$. Тогда справедливо:

a) $L_{d} \simeq \mathbb{Z}^{n} / \mathrm{K}^{*}(F)(\forall d \in \operatorname{ri}(F))$ и все решетки $L_{d} \partial \Omega_{я} d \in \operatorname{ri}(F)$ имеют одну и ту же размерность;

б) размерность решетки $L_{d}$ равна $\operatorname{dim} L_{d}=n-\operatorname{dim} \mathrm{K}^{*}(F)$;

в) все $L$-многогранники $P_{d} \partial л я d \in \operatorname{ri} F$ аффинно эквивалентны.

ДокАЗАТЕЛЬСтво. Для доказательства утверждения б) рассмотрим расширение $\bar{\omega}_{d}$ отображения $\omega_{d}$ в $\mathbb{R}^{n}$ и заметим, что $\operatorname{dim} L_{d}=\operatorname{dim}\left(\operatorname{Im} \bar{\omega}_{d}\right)$. С другой стороны, $\operatorname{dim}\left(\operatorname{Ker} \omega_{d}\right)=\operatorname{dim}\left(\operatorname{Ker} \bar{\omega}_{d}\right)$, поскольку в $\operatorname{Ker} \bar{\omega}_{d}$ можно выбрать целочисленньй базис из $\operatorname{Ker} \omega_{d}$, так как $\operatorname{Ker} \bar{\omega}_{d}$ есть множество решений уравнения

$$
\sum_{i=1}^{n} y^{i} \bar{v}_{i}=\mathbf{0}
$$

относительно $\boldsymbol{y} \in \mathbb{R}^{n}$, которое после выбора основного базиса решетки $L_{d}$ преврашается в систему целочисленных линейных однородных уравнений.

5. Представление граней конуса $C_{n+1}$. Для произвольного подмножества $S \subset$ $X=\{0, \ldots, n\}(S \neq \varnothing, X)$ рассмотрим вектор $c^{S} \in \mathbb{R}^{N}$, ортогональньй сut-метрике $d^{S} \in \mathbb{R}^{N}$ и заданный соотношением $c_{i j}^{S}=0$, если $|\{i, j\} \cap S|=1$, и $c_{i j}^{S}=1$ в противном случае. Очевидные соотношения $c^{S} \cdot d^{S}=0, c^{S_{1}} \cdot d^{S_{2}}>0$, если $S_{1} \neq S_{2}$, показывают, что гиперплоскость $c^{S} \cdot d=0$ в пространстве $\mathbb{R}^{N}$ отделяет cut-вектор $d^{S}$ от всех других cut-векторов. Следовательно, множество cut-векторов в точности соответствует множеству направлений, задающих ребра конуса $C_{n+1}$. 
Рассмотрение сечения $C_{n+1}^{*}=C_{n+1} \cap H$ конуса $C_{n+1}$ гиперплоскостью $H$, заданной уравнением

$$
\sum_{0 \leqslant i<j \leqslant n} d_{i j}=1
$$

и взаимно однозначного соответствия между гранями $C_{n+1}$ и $C_{n+1}^{*}$ позволяет применить для $C_{n+1}$ известные факты теории вьпуклых многогранников.

Множество ребер любой грани $F$ конуса $C_{n+1}$ совпадает с множеством ребер конуса $C_{n+1}$, содержашихся в $F$, причем $F$ является конической оболочкой этого множества. Последнее позволяет в дальнейшем отождествлять любую грань конуса $C_{n+1}$ с содержащимся в ней множеством cut-векторов.

6. Невырожденные грани cut-конуса $C_{n+1}$. Размерность решетки $L_{d}$ для $d \in C_{n+1}$. Пусть $d^{S} \in C_{n+1}$ - cut-метрика, соответствующая множеству $S \subset X=$ $\{0, \ldots, n\}(S \neq \varnothing, X)$, ипусть $\left(y^{0}, \ldots, y^{n}\right),\left(x^{0}, \ldots, x^{n}\right) \in \mathbb{Z}^{n+1},\left(y^{0}, \ldots, y^{n}\right) \neq(0, \ldots, 0)$, $\sum_{0 \leqslant i \leqslant n} y^{i}=0, \sum_{0 \leqslant i \leqslant n} x^{i}=1$. Тогда

$$
\begin{aligned}
\sum_{0 \leqslant i<j \leqslant n} y^{i} y^{j} d_{i j}^{S} & =\sum_{i \in S, j \notin S} y^{i} y^{j} d_{i j}^{S}=-s_{0}^{2}<0, \quad \text { где } s_{0}=\sum_{i \in S} y^{i} \\
\sum_{0 \leqslant i<j \leqslant n} x^{i} x^{j} d_{i j}^{S} & =\sum_{i \in S, j \notin S} x^{i} x^{j} d_{i j}^{S}=s_{1}\left(1-s_{1}\right) \leqslant 0, \quad \text { где } s_{1}=\sum_{i \in S} x^{i} .
\end{aligned}
$$

Пусть $d$ - гиперметрика из относительной внутренности грани $F$ конуса $C_{n+1}$. Рассмотрим линейное отображение $\omega: \mathbb{R}^{n} \rightarrow E^{k}$,

$$
\omega: \boldsymbol{x}=\left(x^{1}, \ldots, x^{n}\right) \mapsto \omega(\boldsymbol{x})=\sum_{1 \leqslant i \leqslant n} x^{i} \bar{v}_{i}
$$

где векторы $\bar{v}_{1}, \ldots, \bar{v}_{n}$ определены в п. 2 для гиперметрики $d$. Можно представить $\omega$ как композицию $\omega=\delta$ о $\gamma\left(\omega: \mathbb{R}^{n} \stackrel{\gamma}{\rightarrow} \mathbb{R}^{n+1} \stackrel{\delta}{\rightarrow} E^{k}\right)$, где $\gamma$ - изоморфизм $\mathbb{R}^{n}$ в подпространство $\mathbb{R}^{n+1}$, определенньй соотношениями

$$
y^{0}=-\sum_{1 \leqslant i \leqslant n} x^{i}, \quad y^{i}=x^{i}, \quad 1 \leqslant i \leqslant n,
$$

a $\delta$ определяется соотношениями

$$
\omega(\boldsymbol{y})=\sum_{0 \leqslant i \leqslant n} y^{i} \bar{v}_{i}, \quad \bar{v}_{0}=\overline{0}
$$

Рассмотрим ядро отображения $\delta$ :

$$
\begin{aligned}
\operatorname{Ker}(\delta) & =\left\{\boldsymbol{y}=\left(y^{0}, \ldots, y^{n}\right) \in \mathbb{R}^{n+1} \mid \sum_{0 \leqslant i \leqslant n} y^{i}=0, \sum_{0 \leqslant i \leqslant n} y^{i} \bar{v}_{i}=\overline{0}\right\} \\
& =\left\{\boldsymbol{y}=\left(y^{0}, \ldots, y^{n}\right) \in \mathbb{R}^{n+1} \mid \sum_{0 \leqslant i \leqslant n} y^{i}=0, \sum_{0 \leqslant i<j \leqslant n} y^{i} y^{j} d_{i j}=0\right\} .
\end{aligned}
$$


Обозначим аналогично тому, как это сделано в п. 2, через

$$
H_{\boldsymbol{y}}=\left\{h=\left(h_{i j}\right)_{0 \leqslant i<j \leqslant n} \in \mathbb{R}^{N} \mid \sum_{0 \leqslant i<j \leqslant n} y^{i} y^{j} h_{i j}=0\right\}
$$

гиперплоскость в $\mathbb{R}^{N}$, определенную для каждого $\boldsymbol{y} \in \mathbb{R}^{n+1} \backslash\{\mathbf{0}\}$ с $\sum_{0 \leqslant i \leqslant n} y^{i}=0$. Плоскость $H_{\boldsymbol{y}}$ проходит через относительно-внутреннюю точку $d \in \mathbb{R}^{N}$ грани $F$ конуса $C_{n+1}$ в том и только том случае, когда $H_{\boldsymbol{y}}$ содержит все ребра конуса $C_{n+1}$, определяющие грань $F$. Другими словами, $\operatorname{Ker}(\delta)$ можно представить как множество решений системы

$$
\sum_{0 \leqslant i \leqslant n} y^{i}=0, \quad \sum_{0 \leqslant i<j \leqslant n} y^{i} y^{j} d_{i j}^{S_{l}}=0, \quad 1 \leqslant l \leqslant r,
$$

где $d^{s_{1}}, \ldots, d^{s_{r}}$ - cut-метрики, определяющие грань $F$. С учетом соотношений $(15)$ система (18) эквивалентна системе

$$
\sum_{0 \leqslant i \leqslant n} y^{i}=0, \quad \sum_{i \in S_{l}} y^{i}=0, \quad 1 \leqslant l \leqslant r
$$

Пусть $q$ - ранг системы (19). Размерность пространства решений есть $(n+1)-q=$ $\operatorname{dim}(\operatorname{Ker} \delta)$. Поскольку $\operatorname{Im}(\gamma) \subset \operatorname{Ker}(\delta)$, то $\operatorname{Ker}(\omega)=\gamma^{-1}(\operatorname{Ker} \delta)$ и $\operatorname{dim}(\operatorname{Ker} \omega)=$ $\operatorname{dim}(\operatorname{Ker} \delta)$. Следовательно, $\operatorname{dim}(\operatorname{Im} \omega)=n-\operatorname{dim}(\operatorname{Ker} \omega)=q-1$, а размерность точечной решетки удовлетворяет соотношению

$$
\operatorname{dim}\left(L_{d}\right)=\operatorname{dim}\left(\omega\left(\mathbb{Z}^{n}\right)\right)=\operatorname{rank}\left\{\bar{v}_{i}\right\}_{1 \leqslant i \leqslant n}=\operatorname{dim}(\operatorname{Im} \omega) .
$$

Таким образом, доказана следующая лемма.

Лемма 4. Размерность точечной решетки, определяемой любой гиперметрикой $d$ из относительной внутренности грани $F$ конуса $C_{n+1}$, равна $q-1$, где $q$ - ранг системы (19), соответствующей грани $F$. В частности, грань $F$ невырождена в том и только том случае, когда система (19) имеет ранг $n+1$ (невырождена).

СлЕдСТВИЕ. Всякая грань $Q$, принадлежащая вырожденной грани $F$ конуса $C_{n+1}$, сама является вырохденной. $B$ частности, если одна из граней $F$ и $G$ конуса $C_{n+1}$ вырождена, то $Q=F \cap G$ вырождена.

7. Матрица $M(F)$ невырожденной грани $F$ конуса $C_{n+1}$. Приведем способ отыскания матрицы $M(F)$ барицентрических координат вершин $L$-многогранника, соответствующего любой из невырожденных граней $F$ cut-конуса $C_{n+1}$. Очевидно, что $M(F)=\left\{\boldsymbol{u}_{i}\right\}_{i=0}^{n} \cup \operatorname{Ann}(F)=\left\{\boldsymbol{u}_{i}\right\}_{i=0}^{n} \cup\left\{\boldsymbol{x} \in \mathbb{Z}^{n+1} \backslash\left\{\boldsymbol{u}_{i}\right\}_{i=0}^{n} \sum_{0 \leqslant i \leqslant n} x^{i}=1, F \subset H_{\boldsymbol{x}}\right\}$.

Рассуждая, как в п. 6, с учетом соотношений $(16)$ заключаем, что $M(F)$ является множеством целочисленных решений системы

$$
\sum_{0 \leqslant i \leqslant n} x^{i}=1, \quad\left(\sum_{i \in S_{l}} x^{i}\right)\left(1-\sum_{i \in S_{l}} x^{i}\right)=0, \quad 1 \leqslant l \leqslant r,
$$


где $d^{S_{1}}, \ldots, d^{S_{r}}$ - cut-метрики, задаюшие грань $F$. Множество решений системы получается объединением решений $2^{r}$ систем

$$
\sum_{0 \leqslant i \leqslant n} x^{i}=1, \quad \sum_{i \in S_{l}} x^{i}=\varepsilon_{l}, \quad 1 \leqslant l \leqslant r, \quad \varepsilon_{l} \in\{0,1\},
$$

каждая из которых в случае невырожденности грани $F$ имеет не более одного решения. (Выделив $n+1$ линейно независимьх строк основной матрищы системы (21), включая первую, можно свести семейство к $2^{n}$ системам, каждая из которых имеет единственное решение, проверяемое затем на принадлежность множеству $\mathbb{Z}^{n}$ и вьполнение оставшихся равенств из системы (20).)

8. Классы эквивалентности граней конуса $C_{n+1}$ и их матричное представление. Пусть $\varphi$ - перестановка множества $X=\{0, \ldots, n\}$ (биекция $X$ в себя). Определим отображения $\varphi^{*}$ и $\bar{\varphi}$ :

$$
\begin{array}{ll}
\varphi^{*}: \mathbb{R}^{N} \rightarrow \mathbb{R}^{N}, & \varphi^{*}: h_{i j} \mapsto \varphi^{*}(h)_{i j}=h_{\varphi(i) \varphi(j)}, \\
\bar{\varphi}: \mathbb{Z}^{n+1} \rightarrow \mathbb{Z}^{n+1}, & \bar{\varphi}:\left(x^{0}, \ldots, x^{n}\right) \mapsto\left(x^{\varphi(0)}, \ldots, x^{\varphi(n)}\right) .
\end{array}
$$

Пусть $F$ и $G$-грани конуса $C_{n+1}$. Будем говорить, что $F \pi$-әквивалентна $G(F \stackrel{\pi}{\sim} G)$, если существует перестановка $\varphi$ множества $X=\{0, \ldots, n\}$, индуцирующая биекцию $\varphi^{*}$ подмножеств cut-метрик, определяющих грани $F$ и $G$. Тогда очевидно, что $\varphi^{*}(F)=G$, где $F$ и $G$ рассматриваются как множества в $\mathbb{R}^{N}$.

Заметим, что

$$
\sum_{0 \leqslant i<j \leqslant n} x^{i} x^{j} h_{\varphi(i) \varphi(j)}=\sum_{0 \leqslant i<j \leqslant n} x^{\varphi^{-1}(i)} x^{\varphi^{-1}(j)} h_{i j} .
$$

Поэтому $\operatorname{Ann}\left(\varphi^{*}\{F\}\right)=\bar{\varphi}^{-1}\{\operatorname{Ann}(F)\}$ и $\mathrm{K}\left(\varphi^{*}\{F\}\right)=\bar{\varphi}^{-1}\{\mathrm{~K}(F)\}$. Следовательно, справедлива следующая лемма.

Лемма 5. Аффинные типы L-многогранников, поставленные в соответствие (леммой 9) $\pi$-эквивалентным граням конуса $C_{n+1}$, совпадают. (В частности, совпадают их размерность и характеристика невырожденности.) Матрицы $M(F)$ и $M(G)$ для $\pi$-әквивалентных невырожденных граней $F$ и $G$ конуса $C_{n+1}$ отличаются лишь расстановкой столбиов: $M\left(\varphi^{*}\{F\}\right)=\bar{\varphi}^{-1}\{M(F)\}$, где $G=\varphi^{*}(F)$.

Из леммы 5 вытекает, что в дальнейшем мы можем говорить о невырожденном $\pi$ классе граней конуса $C_{n+1}$ и аффинном типе $L$-многогранников $\pi$-класса граней конуса $C_{n+1}$.

В дальнейшем для реализации алгоритма нахождения аффинных типов $L$-многогранников важно для каждого класса $\pi$-эквивалентньх граней конуса $C_{n+1}$ зафиксировать такой представитель, который можно восстановить, имея в распоряжении любую грань того же класса, другими словами, иметь алгоритм получения граней канонического вида.

Каждой cut-метрике $d^{S}(S \subset X, S \neq \varnothing)$ сопоставим характеристический столбец $\left(\epsilon^{0}, \ldots, \epsilon^{n}\right)^{T}$, где $\epsilon^{i}=1$, если $i \in S$, и $\epsilon^{i}=0$ в противном случае. Пусть $F-$ произвольная грань конуса $C_{n+1}$. Поставим ей в соответствие матрицу $\widetilde{F}$ (определенную неоднозначно) размера $(n+1) \times\left|F_{c}\right|$, составленную из характеристических столбцов, соответствующих cut-метрикам, входящим в $F$. И пусть $\mathscr{F}$ - множество всевозможных 
матриц $\widetilde{F}$, поставленных в соответствие граням конуса $C_{n+1}$. Рассмотрим преобразования матриц $\widetilde{F}$ на множестве $\mathscr{F}$ :

a) замена в одном из столбцов нулей на единицы, и наоборот;

b) перестановка столбцов;

c) перестановка строк.

Преобразования а), b) сохраняют грань $F$, а преобразование с) сохраняет класс эквивалентности грани $F$. Таким образом, справедлива следуюшая лемма.

ЛЕмма 6. Существуют взаимно однозначные соответствия мехуду:

1) гранями конуса $C_{n+1}$ и классами әквивалентности матрии, $\widetilde{F} \in \mathscr{F}$ относительно преобразований а), b) (называемые далее аb-классами);

2) $\pi$-классами граней конуса $C_{n+1}$ и классами әквивалентности матрии, $\widetilde{F} \in \mathscr{F}$ относительно преобразований a), b), с) (называемые далее аbс-классами).

Внутри каждого ab-класса матриц, представляющего фиксированную грань $F$ конуса $C_{n+1}$, выберем однозначно определенного фиксированного ab-представителя $\widehat{F}$, в котором

$\mathrm{A}_{1}$ ) последняя строка состоит из нулей (преобразования а));

$\mathrm{A}_{2}$ ) столбцы идут в возрастающем порядке (читая их как двоичную запись натурального числа снизу вверх).

Легко видеть, что количество ab-классов (и их представителей $\widehat{F}$ ) не превышает $(n+1)$ !. Каждому ab-представителю $\widetilde{F} \in \mathscr{F}$ поставим в соответствие натуральное число $\chi(\widetilde{F}) \in \mathbb{N}$, двоичная запись которого получается последовательньм присоединением столбцов матрицы $\widetilde{F}$ (читая их снизу вверх, слева направо).

ОПРЕДЕЛЕНИЕ. Канонической матрицей для $\pi$-класса $\left\{F_{\alpha}\right\}$ граней конуса $C_{n+1}$ будем считать матрицу-представителя $\widetilde{F}$ ab-класса (удовлетворяющую условиям $\mathrm{A}_{1}$ ), $\left.\mathrm{A}_{2}\right)$ ), которая имеет наименьшее значение $\chi(\widetilde{F})$ на множестве ab-представителей.

9. Матричное представление аффинных типов $L$-многогранников. Два конечногранных выпуклых $n$-мерных многогранника $P$ и $Q \subset E^{n}$ аффинно әквивалент$\boldsymbol{H b l}$, если существует невырож денное аффинное преобразование $\Psi$ пространства $E^{n}$, осуществляющее биекцию множеств точек многогранников: $P=\Psi(Q)$. Заметим, что для аффинной эквивалентности необходимо и достаточно, чтобы аффинное преобразование $\Psi$ осуществляло биекцию лишь множеств вершин многогранников: $\operatorname{ext} P=\Psi(\operatorname{ext} Q)$.

Очевидна следующая лемма.

ЛЕмма 7. Два многогранника $P$ u $Q \in E^{n}$ аффинно әквивалентны тогда $и$ только тогда, когда среди вершин каждого можно выбрать такой барицентрический базис пространства $E^{n}: V=\left\{V_{0}, \ldots, V_{n}\right\} \subset \operatorname{ext} P, U=\left\{U_{0}, \ldots, U_{n}\right\} \subset \operatorname{ext} Q$, что барицентрические координаты вершин многогранника $P$ в базисе $V$ совпадают с баричентрическими координатами вершин $Q$ в базисе $U$.

Дадим описание приведения матрицы барицентрических координат $L$-многогранника к канонической матрице относительно аффинного типа $L$-многогранников. Ограничим множество рассматриваемых $L$-многогранников только такими, среди вершин которых можно выбрать основной симплекс решетки. Для дальнейшего это ограничение естественно, поскольку мы будем рассматривать только $L$-многогранники, полученные на основе невырожденных гиперметрик. 
Пусть $P$ - один из $L$-многогранников некоторой решетки $L$, и пусть $\mathscr{M}=\left\{M_{V}: V \in \mathscr{V}\right\}$ - множество всевозможных матрищ $M_{V}$ размера $\mid$ ext $P \mid \times(n+1)$, вектор-строки которых являются барищентрическими координатами вершин $P$ в барицентрическом базисе $V$ решетки $L$. Указанное вьше множество $\mathscr{V}$ барицентрических базисов решетки $L$ ограничим основньми симплексами решетки с вершинами, выбранньми среди вершин $P$ и имеющими наибольшее возможное количество гиперграней, лежащих на поверхности многогранника $P$. (Каждая такая грань однозначно соответствует столбцу матрищы $M_{V} \in \mathscr{M}$, не содержащему отрищательных значений.)

Остановимся на способе получения матриц множества $\mathscr{M}$. Пусть $M$ - матрица размера $|\operatorname{ext} P| \times(n+1)$ барицентрических координат вершин $P$ относительно некоторого основного барицентрического базиса $B=\left\{B_{0}, \ldots, B_{n}\right\}$ решетки $L$, т.е.

$$
\begin{gathered}
L=\left\{V \in E^{n}: \overline{O V}=\sum_{\substack{0 \leqslant i \leqslant n \\
\bar{b}_{i}=\overline{O B_{i}}, \quad 0 \leqslant i \leqslant n .}} x^{i} \bar{b}_{i}, \sum_{0 \leqslant i \leqslant n} x^{i}=1,\left(x^{0}, \ldots, x^{n}\right) \in \mathbb{Z}^{n+1}\right\} \\
\end{gathered}
$$

Пусть $V$ - это некоторое подмножество вершин многогранника $P$ с $|V|=n+1$, а $Y_{V}-$ матрица размера $(n+1) \times(n+1)$, составленная из тех строк матрицы $M$, что отвечают множеству вершин $V$. Множество вершин $V$ определяет основной симплекс решетки $L$ в том и только том случае, когда $\operatorname{det}\left(Y_{V}\right)=1$. В этом случае матрица $M_{V}$ барищентрических координат вершин многогранника $P$ имеет вид $M_{V}=M \cdot Y_{V}^{-1}$.

Очевидно, что множество $\mathscr{M}_{V}=\left\{M_{V}\right\}$ описанных выше координатных матрищ в фиксированном базисе $V$ не зависит от выбора первоначального барищентрического базиса $B$ решетки $L$, а сами матрицы $M_{V} \in \mathscr{M}_{V}$ отличаются друг от друга лишш расстановкой строк и столбцов.

Далее введем следующую вспомогательную конструкцию. Для произвольной матрицы $A$ размера $q \times(n+1)$ обозначим через $\Lambda(A)=\left(a_{1}, \ldots, a_{q(n+1)}\right)$ строку, полученную последовательным присоединением столбцов матрицы $M$. Для произвольных матрищ $A, B$ того же размера введем отношение лексической упорядоченности.

ОПРЕДЕЛЕНИЕ. Матрица $A$ лексически меньше матрицы $B(A \prec B)$, если найдется число $l \in \mathbb{Z}, 1 \leqslant l \leqslant q(n+1)$, что для $\Lambda(A)=\left(a_{1}, \ldots, a_{q(n+1)}\right)$ и $\Lambda(B)=\left(b_{1}, \ldots, b_{q(n+1)}\right)$ выполнено

1) $a_{i}=b_{i}(1 \leqslant i<l)$;

2) $a_{l}<b_{l}$.

(Будем считать, что $A \preceq B \Longleftrightarrow[A \prec B$ либо $A=B]$.)

Поскольку $\left|\mathscr{M}_{V}\right| \leqslant(n+1) ! q$ !, то среди матриц из множества $\mathscr{M}_{V}$ (при фиксированном $V \in \mathscr{V})$ всегда существует наименьшая относительно лексической упорядоченности матрица $\bar{M}_{V} \in \mathscr{M}$ (т.е. $\left.\bar{M}_{V} \preceq M_{V} \forall M_{V} \in \mathscr{M}_{V}\right)$. Пусть $\overline{\mathscr{M}}=\left\{\bar{M}_{V}: V \in \mathscr{V}\right\}-$ множество матриц $\bar{M}_{V}$, поставленных индексами $V$ в однозначное соответствие симплексам $V \in \mathcal{V}$. Конечность способов выбора симплекса $V \in \mathcal{V}$ обеспечивает конечность множества $\overline{\mathscr{M}}$.

ОПРЕДЕЛЕНИЕ. Пусть $P$ - это $L$-многогранник решетки $L$, содержащий среди своих вершин хотя бы один основной симплекс решетки $L$. Матрищу $\bar{M}_{V} \in \overline{\mathscr{M}}$, лексически наименьшую на множестве $\mathscr{M}$, будем назьвать канонической относительно аффинного типа $L$-многогранника $P$. 


\section{0. Алгоритм нахождения аффинных типов $L$-многогранников размер-} ности $n \leqslant 5$, содержащих основной симплекс решетки. Результаты. В этом пункте мы будем считать, что для множества $X=\{0, \ldots, n\}$ значение $n \leqslant 5$, и иметь ввиду, что для таких $n$ справедливо равенство $C_{n+1}=H Y P_{n+1}$.

Как следует из леммы 5 , вопрос о нахождении аффинных типов $L$-многогранников, содержащих среди своих вершин основной симплекс решетки, сводится к нахождению всех $\pi$-типов невырожденных граней конуса $C_{n+1}$.

Всюду ниже, говоря о гранях конуса $C_{n+1}$, мы будем иметь в виду множества cut-метрик, содержащихся в этих гранях. Для гиперграней конуса $C_{n+1}=H Y P_{n+1}(n \leqslant 5)$ эти множества получаем из того факта, что каждая гипергрань лежит в некоторой гиперплоскости $H_{\boldsymbol{x}}$, где точка $\boldsymbol{x}=\left(x^{0}, \ldots, x^{n}\right) \in \mathbb{Z}^{n+1}, \sum_{i=0}^{n} x^{i}=1$, принадлежит множеству минимальных заслоняющих точек (см. п. 2).

Используя соотношение (16), получаем, что

$$
F=\left\{d^{S} \mid S \subset X, \sum_{i \in S} x^{i} \in\{0,1\}\right\} .
$$

Так как сечение $C_{n+1}^{*}$ конуса $C_{n+1}$ является выпукльм конечногранньп многогранником, то любая его грань размерности $k-1$ может быть получена пересечением некоторой грани размерности $k$ и некоторой не содержащей ее гиперграни. Эти утверждения переносятся на соответствующие подмножества вершин многогранника $C_{n+1}^{*}$ и cut-метрик, определяющих ребра конуса $C_{n+1}$.

Пусть $\mathscr{E}^{k}=\left\{F_{i}\right\}_{i=1}^{q_{k}}-$ множество граней конуса $C_{n+1}$ размерности $k$, а $\mathscr{E}^{N-1}=$

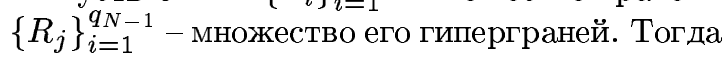

$$
\begin{gathered}
\mathscr{E}^{k-1} \subseteq\left\{Q_{i j}\right\}:= \\
\left\{F_{i} \cap R_{j} \mid F_{i} \nsubseteq R_{j}, 1 \leqslant i \leqslant q_{k}, 1 \leqslant j \leqslant q_{N-1}\right\} \\
\text { для } 2 \leqslant k<N=\left(\begin{array}{c}
n+1 \\
2
\end{array}\right),
\end{gathered}
$$

причем $\operatorname{dim} Q_{i j} \leqslant(k-1)$. Для того чтобы выделить из множества $\left\{Q_{i j}\right\}$ грани размерности $k-1$, достаточно заметить, что размерность грани $Q_{i j}$ равна рангу матрицы размера $\left|Q_{i j}\right| \times n(n+1) / 2$, строки которой соответствуют векторной записи сut-метрик $d^{S}=\left(d_{01}^{S}, \ldots, d_{n-1}^{S}\right)$, содержащихся в $Q_{i j}$. Таким образом, имея семейство гиперграней $\mathscr{E}^{N-1}$ и повторяя $N-2=\left(\begin{array}{c}n+1 \\ 2\end{array}\right)-2$ раза описанную выше процедуру, мы можем найти все грани конуса $C_{n+1}$.

Применение такого алгоритма отыскания граней, запрограмированного для ПК, приемлемо по объему (и времени) вычислений лишш для $n \leqslant 4$. Для $n=5$ потребовались дополнительные соображения, сокращающие вычисления. Суть их заключается в том, чтобы для каждой размерности $k\left(1 \leqslant k \leqslant\left(\begin{array}{c}n+1 \\ 2\end{array}\right)-1\right)$ находить лишш грани множества $\overline{\mathscr{G}}^{k}=\left\{\widehat{F}_{i}\right\}_{i=1}^{q_{k}}$, соответствующего семейству классов $\pi$-эквивалентности невырожденных граней конуса $C_{n+1}$, причем каждый $\pi$-класс представлен в множестве $\overline{\mathscr{G}}^{k}$ невырожденной гранью $\widehat{F}_{i}$, соответствующей канонической матрице $\pi$-типа. Легко видеть, что множество $\mathscr{G}_{k}:=\left\{\varphi^{*}(\widehat{F}) \mid \widehat{F} \in \overline{\mathscr{G}}^{k}, \varphi \in S_{n+1}\right\}$ есть множество всех невырожденных граней размерности $k$. (Напомним, что $\varphi^{*}$ - отображения, определенные в п. 8 и соответствующие всевозможньм перестановкам $\varphi$ множества $X=\{0, \ldots, n\}$.) Поэтому

$$
\overline{\mathscr{G}}^{k-1} \subseteq \overline{\mathscr{Q}}:=\left\{\varphi^{*}\left(\widehat{F}_{i}\right) \widehat{\cap \psi^{*}}\left(\widehat{R}_{j}\right) \mid 1 \leqslant i \leqslant q_{k}, 1 \leqslant j \leqslant q_{N-1}, \varphi, \psi \in S_{n+1}\right\},
$$


где $\left\{\widehat{F}_{i}\right\}_{i=1}^{q_{k}}=\overline{\mathscr{G}}^{k},\left\{\widehat{R}_{i}\right\}_{i=1}^{q_{N-1}}=\overline{\mathscr{G}}^{N-1}$. (Здесь $\widehat{Q}$ означает грань конуса $C_{n+1}, \pi$-эквивалентную грани $Q$ и соответствующую канонической матрице $\pi$-типа.)

Очевидные сами по себе утверждения следующей леммы значительно сокращают представление множества $\overline{\mathscr{Q}}$.

ЛЕмма 8. Для любых граней $F$ и $G$ конуса $C_{n+1}$ и перестановок $\varphi$ и $\psi$ множества $X$ справедливо:

1) $\varphi^{*}(F \cap G)=\varphi^{*}(F) \cap \psi^{*}(G)$;

2) $\varphi^{*}(F) \cap \psi^{*}(G) \stackrel{\pi}{\sim}\left(\psi^{-1} \circ \varphi\right)^{*}(F) \cap G$;

3) если $\left(\varphi^{-1}\right)^{*}(F)=\left(\psi^{-1}\right)^{*}(F)$, mo $F \cap \varphi^{*}(G) \stackrel{\pi}{\sim} F \cap \psi^{*}(G)$.

Утверждение 2) леммы 8 позволяет представить

$$
\overline{\mathscr{G}}^{k-1} \subseteq \overline{\mathscr{Q}}=\left\{\widehat{F}_{i} \widehat{\cap \varphi^{*}}\left(\widehat{R}_{j}\right) \mid 1 \leqslant i \leqslant q_{k}, 1 \leqslant j \leqslant q_{N-1}, \varphi \in S_{n+1}\right\},
$$

а утверждение 3 ) для каждой пары $\widehat{F}_{i} \widehat{R}_{j}$ дает возможность ограничиться лишш таким множеством перестановок $\varphi \in S_{n+1}$, которое определяет попарно различные грани $\varphi^{*}\left(\widehat{R}_{j}\right)\left(1 \leqslant j \leqslant q_{N-1}\right)$ и различные $\left(\varphi^{-1}\right)^{*}\left(\widehat{F}_{i}\right)\left(1<i \leqslant q_{k}\right)$.

Приведем алгоритм нахождения аффинных типов $L$-многогранников, содержащих основной симплекс решетки, при фиксированном $n \leqslant 5$.

I. Составить полный список $F_{1}^{N-1}, \ldots, F_{q_{N-1}}^{N-1}$ представителей невырожденных $\pi$-типов гиперграней конуса $C_{n+1}$. Для этого каждому типу (относительно перестановки элементов вектор-строки) минимальных заслоняющих точек $\left(x^{0}, \ldots, x^{n}\right) \in \mathbb{Z}^{n+1}$, $\sum_{0 \leqslant i \leqslant n} x^{i}=1$, решетки $L$ поставить в соответствие гипергрань $F$ конуса $C_{n+1}$, определенную соотношением (23). Осушествить проверку невырожденности грани $F$ и найти каноническую матрицу $\pi$-класса граней, эквивалентных грани $F$ (см. п. 6,8$)$.

II. Для каждой гиперграни $F_{j}^{N-1}\left(1 \leqslant j \leqslant q_{N-1}\right)$ составить список пар

$$
\left(\varphi_{j l}, \varphi_{j l}^{*}\left(F_{j}^{N-1}\right)\right)
$$

$\left(0 \leqslant l \leqslant r_{j}\right)$, где набор перестановок $\left\{\varphi_{j l}\right\}$ содержит по одному представителю каждого из правых классов смежности относительно стационарной подгруппы грани $F_{j}^{N-1}$ $\left(\left\{\varphi: \varphi^{*}\left(F_{j}^{N-1}\right)=F_{j}^{N-1}\right\}\right)$, а набор $\varphi_{j l}^{*}\left(F_{j}^{N-1}\right)\left(0 \leqslant l \leqslant r_{j}\right)$ соответственно представляет собой класс граней конуса $C_{n+1}, \pi$-эквивалентных грани $F_{j}^{N-1}$. (Будем считать, что перестановка $\varphi_{j 0}^{*}$ тождественная и $\varphi_{j 0}^{*}\left(F_{j}^{N-1}\right)=F_{j}^{N-1}$.)

III. Найти представителей всех типов невырожденных граней размерностей от $N-1$ $=\left(\begin{array}{c}n+1 \\ 2\end{array}\right)-1$ до 1 , совершая последовательно $\left(\begin{array}{c}n+1 \\ 2\end{array}\right)-2$ раз следующую операцию.

Имея список $F_{1}^{m}, F_{2}^{m}, \ldots, F_{q_{m}}^{m}$ представителей $\pi$-типов невырожденных граней размерности $m\left(2 \leqslant m \leqslant\left(\begin{array}{c}n+1 \\ 2\end{array}\right)-1\right)$, найти аналогичньй список для размерности $m-1$. Для этого для каждой пары граней $\left(F_{i}^{m}, F_{j}^{N-1}\right)\left(1 \leqslant i \leqslant q_{m}, 1 \leqslant j \leqslant q_{N-1}\right)$ :

1) из конечной последовательности перестановок $\varphi_{j 0}, \varphi_{j 1}, \ldots, \varphi_{j r_{m}}$, составленной для грани $F_{j}^{N-1}$ в п. II, выбрать максимальную подпоследовательность $\varphi_{j l_{0}}$, $\varphi_{j l_{1}}, \ldots, \varphi_{j l_{k}}(k \leqslant m)$ таких, что $\left(\varphi_{j l_{s}}^{-1}\right)^{*}\left(F_{i}^{m}\right) \neq\left(\varphi_{j l_{t}}^{-1}\right)^{*}\left(F_{i}^{m}\right)$ при $0 \leqslant s<t \leqslant k$;

2 ) составить пересечения $Q_{i j}^{p}=F_{i}^{m} \cap \varphi_{j l_{p}}^{*}\left(F_{j}^{N-1}\right)(0 \leqslant p \leqslant k)$; затем каждую из граней $Q_{i j}^{p}$ :

а) проверить на невырожденность (см. п. 3); 
б) проверить условие $\operatorname{dim}\left(Q_{i j}^{p}\right)=m-1$;

в) при выполнении условий первых двух пунктов найти каноническую матрицу $\pi$-класса граней, эквивалентных грани $Q_{i j}^{p}$ (см. п. 5);

г) добавить грань $\widehat{Q_{i j}^{p}}$, соответствующую канонической матрице, в список $\overline{\mathscr{G}}^{m-1}$ типов невырожденных граней размерности $m-1$.

IV. Используя список представителей всех невырожденных $\pi$-типов граней конуса $C_{n+1}=H Y P_{n+1}(n \leqslant 5)$, найти список аффинных типов $n$-мерньх $L$-многогранников, выполняя для каждой грани из списка действия:

1) получить матрицы $M$ барицентрических координат вершин $L$-многогранника (см. п. 4);

2) привести матрицы $M$ к каноническому виду относительно аффинного типа $L$-многогранников (см. п. 9).

Конец алгоритма.

Чтобы привести здесь результаты проведения алгоритма, напомним свойство $L$-многогранников, назьваемое слойностью [14]. Пусть $P-n$-мерный $L$-многогранник решетки $L^{n}, F$ - одна из его гиперграней, aff $F$ - гиперплоскость, на которой расположена грань $F, L_{0}^{n-1}=L^{n} \cap$ aff $F-(n-1)$-мерная подрешетка решетки $L^{n}$ (слой). Решетку $L^{n}$ рассматриваем как объединение слоев $L_{k}^{n-1}=L_{0}^{n-1}+k \bar{v}$, где $k$ (номер слоя) пробегает множество $\mathbb{Z}$ целых чисел, а $\bar{v}$ - один из векторов решетки, соединяющий два соседних слоя. Предполагая, что многогранник $P$ расположен по ту сторону гиперплоскости aff $F$, где $k>0$, слойностью многогранника $P$ относительно грани $F$ назьвается максимальньй номер слоя, в котором еще имеются вершины многогранника. Максимальное значение слойностей относительно гиперграней многогранника назьвается слойностью многогранника.

Используемое в таблище понятие ранга $n$-мерного решетчатого $L$-многогранника $P$ означает размерность $\operatorname{dim} F(d)$ наименьшей грани $F(d)$ конуса $H Y P_{m+1}$, содержащей гиперметрику $d \in H Y P_{m+1}$, где $d$ - произвольная гиперметрика на множестве $X=$ $\{0, \ldots, m\}(m \geqslant n+1)$, порождающая данный $L$-многогранник $P$ (с точностью до движения). Поскольку невырожденные аффинные преобразования, сохраняющие аффинный тип $L$-многогранника $P$, сохраняют грань $F(d)$, имеет смысл понятие ранга аффинного типа $L$-многогранника (подробнее см. [7]).

Результаты проведения алгоритма для $n \leqslant 4$ согласуются с хорошо известными данньми, а для $n=5$ приведены в таблице 1 , где столбцы, занумерованные числами от 1 до 4, содержат следующую информацию: 1 - номер аффинного типа (звездочками над номерами помечены центрально-симметричные $L$-многогранники); 2 - количество вершин; 3 - количество гиперграней; 4 - ранг $L$-многогранника; 5 - слойность $L$-многогранника.

При указании гиперграней $L$-многогранников в таблице 1 были использованы обозначения: $S^{n}, P^{n}, C^{n}$ - соответственно $n$-мерный симплекс, параллелепипед, кроссполиэдр (ортаэдр), а также $C^{1} \times Q, p(Q), b p(Q)$ - соответственно призма, пирамида и бипирамида над $(n-1)$-мерным многогранником $Q$. Для оставшихся типов 4 -мерных $L$-многогранников обозначения $S_{4}, S_{5}, S_{6}, S_{8}, T_{6}, S_{11}$ заимствованы из работы [3]. Им соответствуют обозначения: $F_{2}^{7}, F_{2}^{6}, F_{3}^{6}, F_{4}^{5}, F_{5}^{5}, F_{4}^{4}$ в работе [2] и обозначения $T_{7}, T_{8}$, $b p\left[R\left(S^{2}\right)\right], T_{9}, T_{9}^{*}, T_{10}$ в работе [4]. Описание этих многогранников читатель может найти в любой из названных работ [2]-[4]. Вычисления велись на ПК IBM Pentium 200 и потребовали около 12 часов машинного времени. 
11. Доказательство теоремы 1. Известно, что для $n \leqslant 4$ все $n$-мерные $L$-многогранники решеток однослойные, а для $n=5$ не более чем двуслойные (см. [4]). Следовательно, любой $L$-многогранник размерности $n \leqslant 4$, а также любой 5 -мерньй $L$-многогранник, содержащий хотя бы одну однослойную гипергрань, содержит среди своих вершин основной симплекс решетки. Таким образом, при $n \leqslant 4$ метод дает полньй список аффинных типов $L$-многогранников, а при $n=5$ приведенная таблица содержит по крайней мере все аффиинные типы однослойных 5-мерных $L$-многогранников.

Рассмотрение результатов в таблице 1 показывает также, что там содержатся и все двуслойные 5 -мерные $L$-многогранники (перечисленные ранее в [5]), за исключениенм $L$-симплекса двойного объема. Таким образом, получен полньй список всех аффинных типов 5-мерных $L$-многогранников.

Автор пользуется случаем поблагодарить проф. В. П. Гришухина и своего научного руководителя проф. Е.П.Барановского за внимание и помошь при подготовке статьи к печати.

\section{СПИСОК ЦИТИРОВАННОЙ ЛИТЕРАТУРЫ}

[1] Делоне Б. Н. Геометрия положительных квадратичных форм // УМН. 1937. № 3. Р. 16-62; 1938. № 4. P. 102-164.

[2] Erdahl R. M., Ryshkov S.S. The empty sphere // Can. J. Math. 1987. V. 39.№ 4. P. 794-824.

[3] Барановский Е. П., Власов Е. В., Новикова Н. В. Строение $L$-разбиений четырехмерных решеток // Деп. ВИНИТИ. 1991. №78-В91. С. 1-38.

[4] Барановский Е.П., Кононенко П.Г. Об одном способе вывода $L$-многогранников $n$-мерных решеток // Матем. заметки. 2000. Т. 68. №6. С. 830-841.

[5] Барановский Е.П., Кононенко П.Г. Двухслойные $L$-многогранники 5 -мерных решеток // Деп. ВИНИТИ. 1998. № 387-В98. С. 1-28.

[6] Assouad P. Sur les inegalités valides dans $L^{1} / /$ European J. Combin. 1984. V. 5. P. 99-112.

[7] Deza M., Grishukhin V.P., Laurent M. Extreme hypermetric and $L$-polytopes // Colloq. Math. Soc. Janos Bolyai. Budapest. 1991. V. 60. P. 157-209.

[8] Деза М. (Тылкин М. Е.) О геометрии Хэмминга единичных кубов // Докл. АН СССР. 1960. Т. 134. № 5. C. 1037-1040.

[9] Deza M., Grishukhin V.P., Laurent M. The hypermetric cone is polyhedral // Combinatorica. 1993. V. 13(4). P. 397-411.

[10] Deza M., Grishukhin V.P., Laurent M. Hypermetrics in Geometry of Numbers // DIMACS Ser. in Disc. Math. and Theor. Comp. Sci. 1995. V. 20. P. 1-109.

[11] Avis D., Mutt. All facets of the six-point Hamming cone // European J. Combin. 1989. V. 10. № 4. P. 309-312.

[12] Барановский Е. П. Симплексы $L$-разбиений евклидовых пространств // Матем. заметки. 1971. T. 10. №6. С. 659-670.

[13] Барановский Е. П. Об $L$-симплексах шестимерных решеток // Вторая международная конференция "Алгебраические, вероятностные, геометрические, комбинаторные и функционалњные методы в теории чисел" (25-30 сентября 1995г., Воронеж). С. 13.

[14] Рышков С. С., Эрдал Р. М. Поэтажное построение $L$-тел решеток // УМН. 1989. Т. 44. № 2. C. 241-242. 